Chapter 19

\title{
Chemotherapeutic Agent for Glioma
}

\author{
Shinji Kohsaka and Shinya Tanaka \\ Additional information is available at the end of the chapter \\ http://dx.doi.org/10.5772/54353
}

\section{Introduction}

Glioma is the most common primary tumors of the central nervous system, accounting approximately for $30 \%$ of entire CNS tumors, and classified into four clinical grades as I to IV. The most aggressive and lethal tumors is glioblastoma multiforme (GBM) with median survival of only 14.6 months, mainly because of limited effects of conventional post-surgical chemotherapeutic agents and irradiation [1]. In this chapter, we summarize chemotherapeutic agents for glioma focusing on their mechanism of anti-tumor action and the acquisition of resistance to the agents.

\section{Temozolomide}

\subsection{Mechanism of action}

Temozolomide (TMZ) is an alkylating agent which is applied to the treatment of malignant glioma including GBM. TMZ induces DNA methylation of guanine at $\mathrm{O}^{6}$ position (O6-MG; $6 \%$ of adducts formed), as well as 7-methylguanine (N7-MG; 70\% of adducts formed), and 3methyladenine (N3-MA; 9\% of adducts formed) [2]. O6-MG incorrectly pairs with thymine and triggers the mismatch repair (MMR) system leading to double strand break of the genome that result in the arrest of cell cycle and induction of apoptosis. N7-MG and N3-MA are removed by the methylpurine glycosylase followed by AP endonuclease which are the first two enzymes in the base excision repair (BER) pathway. Efficient BER system functions and repairs DNA lesions in normal and tumor cells. 573 patients with newly diagnosed as GBM were randomly assigned to be treated by radiotherapy alone or by radiotherapy plus continuous daily medication of temozolomide [3]. At a median follow-up of 28 months, the median survival was 14.6 months with radiotherapy plus temozolomide and 12.1 months 
with radiotherapy alone. The unadjusted hazard ratio for death in the radiotherapy-plus-temozolomide group was 0.63 (95 percent confidence interval, 0.52 to $0.75 ; \mathrm{P}<0.001$ by the logrank test). The two-year survival rate was 26.5 percent with radiotherapy plus temozolomide and 10.4 percent with radiotherapy alone.

\subsection{MGMT - a key molecule for TMZ resistance}

MGMT specifically removes the methyl/alkyl group from the $\mathrm{O}^{6}$-position of guanine and restore the guanine to its normal form escaping from DNA strand breaks (Fig. 1). Thus, the expression of MGMT in tumors has a protective effect against alkylating agents-dependent cell death correlating between MGMT activity and TMZ resistance. MGMT expressing tumor cells exhibit 4- to 10-folds increase of resistance to TMZ, BCNU, and their related compounds [4]. MGMT-mediated repair is unique compared with other DNA repair pathways because : (a) it acts alone without relying on any other proteins or cofactors; (b) it transfers the alkyl group to an internal cysteine residue in the protein, acting as both a transferase and an acceptor of the alkyl-group; (c) it inactivates itself after receiving the alkyl-group from guanine, and thus, it is a suicidal protein; (d) it repairs in a stoichiometric fashion. As one molecule of MGMT removes one alkyl molecule, an excess of DNA adducts at the $\mathrm{O}^{6}$-position could completely deplete MGMT. MGMT is ubiquitously expressed in normal human tissues [5] but is overexpressed in all types of human tumors, including colon cancer, glioma, lung cancer, breast cancer, leukemia, lymphomas, and myeloma. These properties make MGMT as an important drug resistance factor and an ideal target for suppression of drug resistance [2].

\subsection{Regulation of MGMT}

\subsubsection{Promoter methylation}

It is well known that MGMT expression levels vary widely in tumor cells [6; 7]. Hypermethylation of $\mathrm{CpG}$ islands within the promoter region is associated with epigenetic inactivation of the MGMT. In the EORTC trial with 206 GBM patients, MGMT promoter methylation was observed in $45 \%$ cases [8]. In cases with methylated MGMT promoter which means negative of MGMT expression, TMZ was effective as median survival was 21.7 months treated with TMZ and RT compared with 15.3 months with only RT $(\mathrm{P}=0.007)$. A study of German Glioma Network (GGN) also showed that MGMT promoter methylation was associated with prolonged progression-free survival (PFS) and OS in patients receiving TMZ [9]. Several other studies have also shown predictive and prognostic significance of MGMT promoter methylation in GBM [10].

\subsubsection{Transcriptional regulation}

In the MGMT promoter region, there are several specific sequences for the binding of transcription factors including SP1, GRE, AP-1, and NF- $\kappa \mathrm{B}$, thus MGMT can be induced by glucocorticoids, cyclic AMP, protein kinase C activators, and NF- $\kappa B$ [11; 12; 13; 14]. p53 is also reported to suppress MGMT expression by directly binding to the MGMT or by suppressing the transcription factor of SP1 [15; 16]. In addition, MGMT expression can be induced by ra- 
diation or other forms of DNA damages [17]. However, physiological roles and regulation of MGMT induction is not elucidated.

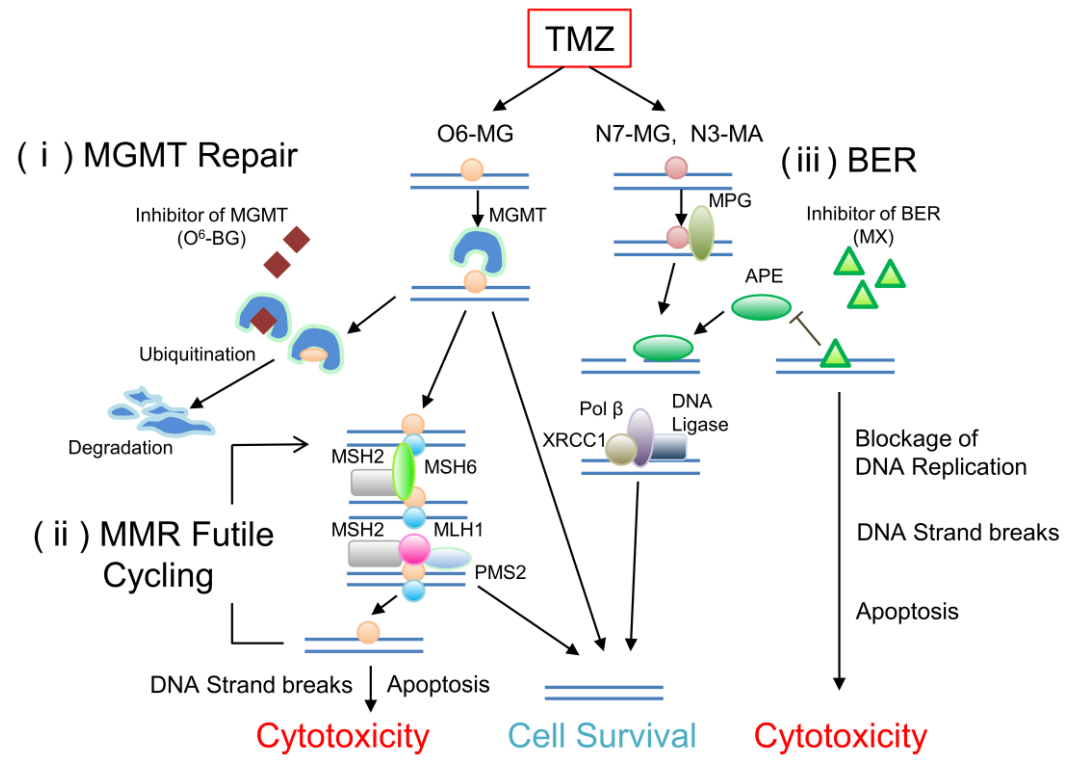

Figure 1. MGMT and other DNA repair mechanisms deal with DNA damage produced by the methylating therapeutic drug, temozolomide (TMZ), in human cells. TMZ and related drugs cause potentially cytotoxic DNA lesions such as O6-methylguanine (O6-MG, orange circle) and N7-methylguanine (N7-MG, brown circle). (i), MGMT (O6-MG DNA methyltransferase) removes the O6-alkylguanine DNA adduct through covalent transfer of the alkyl group to the conserved active-site cysteine and restores the guanine to normal. After receiving a methyl-group from O6-MG, MGMT is inactivated, and subject to ubiquitin-mediated degradation. A similar suicidal enzyme reaction occurs when MGMT transfers and accepts an alkyl-group from O6-benzylguanine (O6-BG), a therapeutic strategies. (ii), if an O6-MG DNA adduct escapes MGMT repair, it would form a base pair with thymine (blue circle) during DNA replication. The mismatched base pair of the persistent O6-MG with thymine is recognized by the mismatch repair pathway, resulting in futile cycles of repair leading to cell death. (iii), N7-MG DNA adducts ( > 70\% of total DNA adducts formed by TMZ) are efficiently repaired by the base excision repair (BER) pathway, and normally they contribute little to the cytotoxicity of TMZ. Methoxyamine binds to AP sites produced by methylpurine glycosylase (MPG), the first step in BER processing. Methoxyamine-bound AP sites are refractory to AP endonuclease (APE, green circle) cleavage, resulting in the blockage of the BER pathway. This leads to strand breaks, disrupted replication, and increased cytotoxicity of TMZ. Figure 1 is adapted from L. Liu et al. Clin Cancer Res. 2006;12(2):328-331.

\subsubsection{Post-transcriptional regulation}

MGMT protein was reported to be degraded via the ubiquitin proteolytic pathway [18]. According to the recent study, the correlation between MGMT promoter methylation and MGMT protein expression was poor $(\mathrm{p}=0.27)$ [19]. In silico analysis predicted potential binding sites for several miRNAs within the 3'UTR of MGMT, suggesting a mechanism for post-transcriptional regulation of MGMT. 


\subsection{Candidate drugs for combination with TMZ}

Strategies to potentiate the effecacy of TMZ by suppressing MGMT or BER pathway have been examined. Pseudosubstrates of MGMT such as $\mathrm{O}^{6}$-benzylguanine were expected to suppress drug resistance by depleting MGMT [20; 21; 22]. However, clinical trials did not show significant restoration of TMZ sensitivity in patients with TMZ-resistant GBM [23]. IFN- $\beta$ down-regulates the expression of MGMT and sensitizes resistant glioma to TMZ and phase II study has been started [15; 24].

We discovered post-transcriptional regulation of MGMT by signal transducer and activator of transcription-3 (STAT3) and demonstrated that STAT3 inhibitor or STAT3 knockdown potentiated TMZ efficacy in TMZ-resistant GBM cell lines [25] (Fig 2). Furthermore, immunohistochemical analysis of 44 malignant glioma specimens demonstrated significant positive correlation between expression levels of MGMT and phosphorylated STAT3 (pSTAT3) $(\mathrm{p}<0.001, \mathrm{r}=0.58)$ (Fig 2). Therefore, STAT3 inhibitor might be one of the candidate reagents for combination therapy with TMZ for TMZ-resistant GBM patients.

\subsection{Other molecules involving TMZ resistance}

In spite of the correlation between promoter methylation of MGMT and temozolomide sensitivity, survival time of the patients who have methylated promoters of MGMT is still short and this suggests the involvement of other mechanism in TMZ resistance. Especially, key molecules of MMR, BER, and Fanconi anemia repair pathway such as MSH6 [26; 27], Nmethyl purine DNA glycosylase (MPG) [28], DNA polymerase $\beta(\operatorname{Pol} \beta)$ [28], alkylpurineDNA-N-glycosylase (APNG) [29] and FANCD1/BRCA2 [30] have been reported to affect to TMZ resistance. The unfolded protein response regulator GRP78/BiP was shown to act as a novel target for increased chemosensitivity in malignant gliomas [31]. Inhibition of Y-box binding protein-1 (YB-1) slows the increased growth of GBM and sensitizes to temozolomide independent of MGMT [32]. High levels of HOXA9/HOXA10 gene expression were associated with a shorter survival in pediatric high-grade glioma patient samples. [33]. Phosphatase and tensin homologue (PTEN) deficiency in GBM confers resistance to radiation and temozolomide that is reversed by the protease inhibitor nelfinavir [34].

\begin{tabular}{|l|l|l|}
\hline Agent & Mechanism of Action & References \\
\hline O6-benzylguanine & $\begin{array}{l}\text { pseudo-substrate of } \\
\text { MGMT }\end{array}$ & $\begin{array}{l}\text { Quinn JA, et al. J Clin Oncol. 27: } \\
1262-1267,2009 .\end{array}$ \\
\hline Interferon- $\beta$ & MGMT inhibition & $\begin{array}{l}\text { Natsume A, et al. Cancer Res. 65: } \\
7573-7579,2005 .\end{array}$ \\
\hline STAT3 inhibitor & MGMT inhibition & $\begin{array}{l}\text { Kohsaka S, et al. Mol Cancer Ther. } \\
\text { In press. }\end{array}$ \\
\hline Levetiracetam & MGMT inhibition & $\begin{array}{l}\text { Bobustuc GC, et al. NeuroOncol. } \\
12: 917-927,2010 .\end{array}$ \\
\hline PARP inhibitor(ABT888) & BER pathway inhibition & $\begin{array}{l}\text { Palma JP, et al. Clin Cancer Res. } \\
15: 7277-7290,2009 .\end{array}$ \\
\hline Methoxyamine & BER pathway inhibition & $\begin{array}{l}\text { Yan L, et al. Clin Cancer Res. } \\
13: 1532-1539,2007 .\end{array}$ \\
\hline
\end{tabular}

Table 1. Candidate drugs for combiunation with TMZ. STAT3 indicates signal transducer and activator of transcription-3; PARP, poly(ADP-ribose) polymerase; BER, base excision repair. 


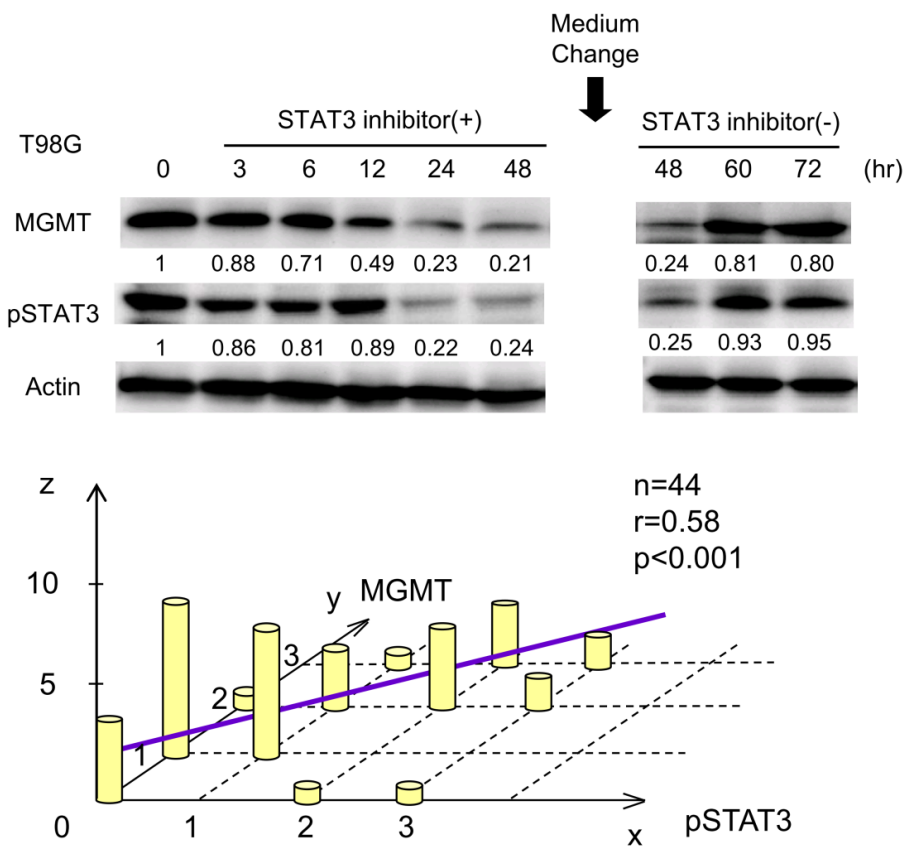

Figure 2. Correlation between expression levels of MGMT and phosphorylated STAT3. (A) Immunoblot analysis of MGMT in T98G treated with 200 mM of STAT3 inhibitor VI. Duration of the treatment is indicated at the top as 0 to 48hr. Medium change indicated removal of STAT3 inhibitor. The level of pSTAT3 was also evaluated (middle panel). Actin is shown as a loading control (bottom panel). (B) Correlation between pSTAT3 and MGMT in 44 cases of malignant glioma specimens. $x$ and $y$ axes indicate score of positivity of pSTAT3 and MGMT, respectively. $z$ axis indicates the number of cases. $n=44$, correlation coefficient $r=0.58, p<0.001$. (A) Immunoblot analysis of MGMT in T98G treated with $200 \mathrm{mM}$ of STAT3 inhibitor VI. Duration of the treatment is indicated at the top as 0 to $48 \mathrm{hr}$. Medium change indicated removal of STAT3 inhibitor. The level of PSTAT3 was also evaluated (middle panel). Actin is shown as a loading control (bottom panel). (B) Correlation between PSTAT3 and MGMT in 44 cases of malignant glioma specimens. $x$ and $y$ axes indicate score of positivity of pSTAT3 and MGMT, respectively. $z$ axis indicates the number of cases. $n=44$, correlation coefficient $r=0.58, p<0.001$.

\section{Targeted molecular agents}

\subsection{Therapeutic targets in GBM}

Identification of biological mechanisms contributing to GBM oncogenesis contributes to provide appropriate targeted therapies to improve patient outcomes. In a large-scale multidimensional analysis performed by the Cancer Genome Atlas involving, the most frequent gene amplifications were: epidermal growth factor receptor (EGFR) and platelet-derived growth factor receptor $\alpha$ (PDGFR $\alpha), 2$ transmembrane receptors with tyrosine kinase activity; cyclin-dependent kinase 4 (CDK4), and murine double minute (MDM)2 and MDM4 which are suppressors for p53 [35]. The most frequent homozygous gene deletions were 
CDKN2A, CDKN2B, and CDKN2C, which encode tumor suppressor proteins that suppress CDK4 and CDK6, phosphatase and tensin homolog (PTEN), a tumor suppressor that inhibits phosphatidylinositol-3 kinase (PI3K) signaling such as retinoblastoma (RB1), a cell-cycle inhibitor as PARK2, a regulator of dopaminergic cell death, and neurofibromin 1 (NF1), a negative regulator of the RAS signal transduction pathway. The most frequently mutated genes were p53, PTEN, NF1, EGFR, human epidermal growth factor receptor 2 (HER2), RB1, and PIK3R1 and PIK3CA-2 components/regulators of the PI3K signaling pathway. This study shows that several genes encoding proteins which are involved in signaling pathways of receptor tyrosine kinases/PI3K, and p53 and the cyclin/RB1, are considerably altered in GBM (Fig. 3). Another study has identified characteristic mutations in the active site of isocitrate dehydrogenase 1 (IDH1) in 12\% of patients with GBM. IDH1 mutations occurred in a high proportion of young patients and in the majority of secondary GBM cases and were associated with increased OS (3.8 years), compared with wild-type IDH1 (1.1 years) [36]. This may be due to increased tumor sensitivity to chemotherapy, although a large controlled series in the German Glioma Network did not find any association between prolonged survival of patients with tumors with IDH1 mutations and administration of a specific therapy [9]. Mutation of the IDH1 active site prevents conversion of isocitrate to $\alpha$-ketoglutarate but allows the mutated enzyme to catalyze the nicotinamide dinucleotide phosphate-dependent reduction of $\alpha$-ketoglutarate to R(-)-2-hydroxyglutarate (2HG) [37]. Accumulated 2HG appears to act as an oncometabolite that contributes to glioma formation and malignant progression. This observation is supported by data from patients with inherited 2hydroxyglutaric aciduria, in whom deficient 2HG dehydrogenase causes an accumulation of brain $2 \mathrm{HG}$. These patients have an increased risk of developing brain tumors, possibly because of increased production of reactive oxygen species [38].

\subsubsection{EGFR}

EGFR is one of the most attractive therapeutic targets in GBM. Approximately $50 \%$ of GBM overexpress EGFR and 25\% express a constitutively active mutated form of EGFR known as EGFRvIII, which has a large deletion in the extracellular domain and renders the receptor ligand independent for signaling [39]. Overexpression of EGFR is more common in primary tumors than in secondary GBM [40]. The deletion also renders a unique codon, which is not found in the wild-type receptor, thereby creating a tumor-specific epitope that can be exploited for therapeutic targeting. Increased EGFR signaling drives tumor cell proliferation, invasiveness, motility, angiogenesis, and inhibition of apoptosis.

\subsubsection{Gefitinib, Erlotinib, Lapatinib and Cetuximab}

Small-molecules of EGFR inhibitor such as gefitinib and erlotinib are well tolerated in patients with malignant gliomas, phase II trials have so far shown limited clinical benefit of erlotinib in patients with either recurrent or newly diagnosed GBM, either in combination regimens $[41 ; 42 ; 43 ; 44]$ or as monotherapy [45]. Neither the EGFR/HER-2 inhibitor lapatinib [46], nor the monoclonal antibody against EGFR, cetuximab [47], have proven to be effective. Attempts to identify biomarkers to predict response to EGFR inhibitors have yielded 


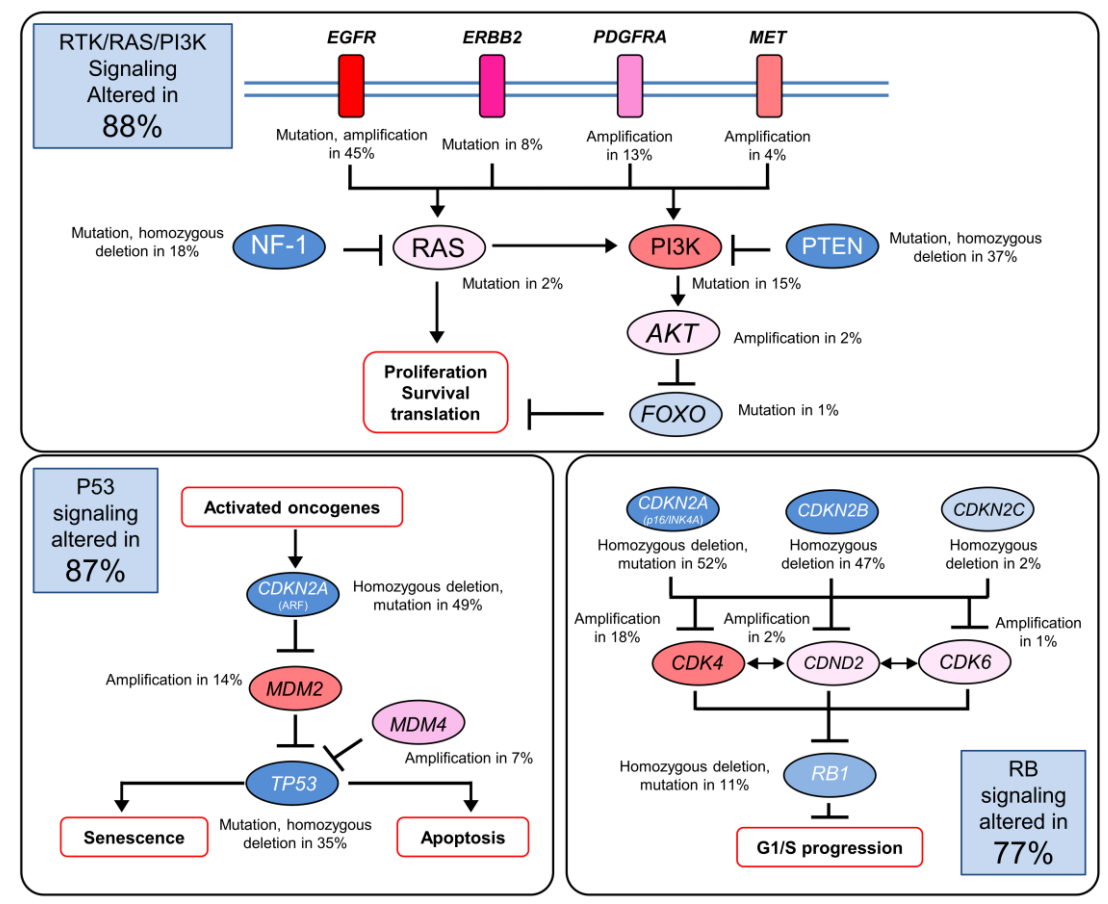

Figure 3. Genetic Alterations in Glioblastoma Occur Frequently in 3 Cellular Signaling Pathways. DNA alterations and copy number changes in the following signaling pathways are indicated in (a) receptor tyrosine kinase (RTK), RAS, and phosphoinositol-3-kinase (PI3K); (b) p53 tumor suppressor; and (c)retinoblastoma (Rb) tumor suppressor. Activating genetic alterations are shown in red. Genetic alterations that lead to a loss of function are indicated in blue. In each pathway, the altered components, the type of alteration, and the percentage of tumors carrying each alteration are shown. Blue boxes contain the total percentages of glioblastomas with alterations in at least 1 known component gene of the designated pathway. Figure 3 is adapted from The Cancer Genome Atlas Research Network. Comprehensive genomic characterization defines human glioblastoma genes and core pathways. Nature 2008;455(7216):1061-1068.

conflicting results. There is no convincing evidence of a correlation between the drug efficacy and the expression levels of EGFR in tumor tissue. In a phase I study, patients with gliomas expressing high levels of EGFR and low levels of activated AKT had better responses to erlotinib than did those with low EGFR expression and high levels of activated AKT [48]. Another study have shown significant correlation of therapeutic response of erlotinib and the presence of EGFR deletion mutant variant III [49]. However, not all studies confirmed these initial observations to predict the sensitivity to EGFR inhibitors [45].

\subsubsection{PDGFR}

PDGFR is a receptor tyrosine kinase with $\alpha$ and $\beta$ isoforms. Overexpression of PDGFR $\alpha$ has been demonstrated in astrocytoma and GBM, indicating a potential role in tumor develop- 
ment [50]. Several PDGFR-targeting agents have been developed that may have therapeutic potential against tumors with elevated PDGFR expression.

\subsubsection{Sorafenib, Imatinib and Tandutinib}

Sorafenib is an orally available antiangiogenic agent that inhibits tumor cell growth and proliferation by blocking the action of intracellular and receptor kinases, including PDGFR, RAF kinase, VEGFR2, and c-KIT [51]. In human GBM cell lines, sorafenib inhibited proliferation synergistically in combination with bortezomib, a proteosome inhibitor [52], and rottlerin, an experimental inhibitor of protein kinase C [53]. A phase II trial found that first-line TMZ and radiotherapy followed by TMZ plus sorafenib was tolerated by patients with GBM, although preliminary efficacy data for this regimen (median PFS duration, 6 months; 12-month PFS rate, $16 \%$ ) were similar to data for standard therapy.

Imatinib mesylate, a small-molecule inhibitor for PDGFR, ABL, and c-KIT, was reported to have significant antitumor activity both in vitro and in vivo as orthotopic glioma models (Kilic et al 2000). Especially, preclinical trials suggested that Imatinib have shows growth inhibition in a subpopulation of CXCL12-expressing GBM cells [54] and radiosensitizes them [55]. However, in phase II trials involving recurrent GBM, imatinib alone or combined with hydroxyurea had limited antitumor activity $[56 ; 57 ; 58 ; 59 ; 60]$.

Tandutinib is an orally active inhibitor of PDGFR, FLT3, and c-KIT tyrosine kinase activity. Although no preclinical data was available for tandutinib in GBM, 2 early-phase trials are assessing tandutinib in recurrent/progressive GBM as monotherapy or combined with bevacizumab. As correlation between increased gene expression levels of PDGFR and preclinical data for therapeutic efficacy was reported, PDGFR may be a promising target for treating GBM. However, the available clinical data suggest otherwise. Trial data of combination regimens involving PDGFR inhibitors are awaited [61].

\subsubsection{VEGFR}

There are multiple reasons for adapting anti-angiogenic drugs to the treatment of malignant gliomas. Malignant glioma exhibits higher vascularization which is one of the pathological hallmarks of GBM. One of the difficulties of developing effective treatments for gliomas has been poor drug penetration through the blood-brain barrier. The dense network of angiogenic vessels in GBM typically display structural, functional, and biochemical abnormalities, including large endothelial cell fenestrae, deficient basement membrane, decreased pericytes and smooth muscle cells, haphazard interconnections with saccular blind-ended extensions, complex tortuosity, and dysregulated transport pathways [62;63; 64; 65; 66; 67]. Therefore, by targeting the tumor vasculature, it is possible to bypass this dependence on drugs to pass the blood-brain barrier to reach their targets. Further, there is also both experimental [68] and clinical [69; 70] evidence that anti-angiogenic drugs can decrease vasogenic edema and patients' requirement for corticosteroids which contributes to morbidity in this population.

The VEGF family of growth factors and their respective receptors are the best characterized proangiogenic proteins in glioma. The VEGF family includes 6 secreted glycoproteins 
(VEGF-A, VEGF-B, VEGF-C, VEGF-D, VEGF-E, and placenta growth factor [PlGF]). VEGF$\mathrm{A}$, the best characterized member, typically localizes adjacent to pseudopalisading necrosis in GBM [71], and the levels of VEGF-A is increased in higher grade of glioma [72; 73], and is associated with poor prognosis [74]. The VEGF receptor (VEGFR) family includes VEGFR-1 (Flt-1), VEGFR-2 (KDR), VEGFR-3, neuropilin-1 (NRP-1), and NRP-2, which exhibit different binding affinities of the VEGF homologs. VEGFR-1 and VEGFR-2 regulate angiogenesis, whereas VEGFR-3 regulates lymphangiogenesis. Vascular endothelial growth factor production and secretion by tumor cells is stimulated mainly by hypoxia, and malignant gliomas are rapidly growing and innately hypoxic tumors. More specifically, VEGF-A binds to VEGFR-2 expressed in blood vessels, which promotes endothelial cell migration and proliferation results in new blood vessel formation in a manner of paracrine signaling loop.

\subsubsection{Bevacizumab}

Bevacizumab, a recombinant humanized monoclonal antibody composed of human immunoglobulin G1 (IgG1; 93\%) and murine VEGF-binding complementarity-determining regions (7\%), binds all isoforms of VEGF with high affinity and specificity [75]. Despite initial reluctance to evaluate bevacizumab in patients with brain tumors owing to concerns of intracranial hemorrhage, a series of 29 patients with recurrent malignant glioma treated with bevacizumab and irinotecan showed no significant hemorrhage with remarkable tumor regression as radiographic response rate of $66 \%$ compared with ordinal chemotherapeutic reagents as rates of $9 \%[76 ; 77]$. These results led rigorous prospective clinical trials of bevacizumab in recurrent malignant gliomas. The combination of bevacizumab and irinotecan was studied in single-arm phase 2 trials for recurrent malignant glioma $(n=33)$ and GBM ( $\mathrm{n}=35)$, showing response rates as $61 \%$ and $57 \%$, and progression-free survival (PFS) at 6 months as $55 \%$ and $46 \%$ [78; 79], respectively. These results were compared with previous rates of PFS at 6 months as $9 \%$ to $15 \%$ for recurrent GBM and $17 \%$ to $31 \%$ for recurrent malignant gliomas [80]. A large phase 2 trial randomized 167 patients of recurrent GBM to analyze efficacy of combination of either bevacizumab or bevacizumab with irinotecan. This noncomparative randomized study showed radiographic response rates as $28 \%$ and $38 \%$, and a PFS at 6 months of $43 \%$ and 50\%, respectively [69]. In addition, patients treated with bevacizumab often exhibit less vasogenic edema and decreased corticosteroid dependence secondary to neutralization of VEGF, a known vascular permeability factor. Another phase 2 trial involved bevacizumab monotherapy in 48 heavily pretreated patients with recurrent GBM [70]. The radiographic response rate was 35\%, and the PFS6 rate was 29\%. Ongoing phase 3 studies are evaluating the combination of bevacizumab with temozolomide and radiotherapy. The results will be of great interest because of the uncertainty regarding the impact of bevacizumab on overall survival. Combinations of bevacizumab and other chemotherapeutics or targeted molecular drugs are also currently in clinical trials.

\subsubsection{Aflibercept}

VEGF Trap (aflibercept) sequesters all isoforms of VEGF-A and PDGF as a soluble, recombinant, decoy receptor, composed of the second Ig domain of VEGFR-1 and the third Ig do- 
main of VEGFR-2 bound to the hinge region of the Fc portion of human IgG1 [81]. Single arm phase II study of aflibercept in recurrent malignant glioma was proceeded [82]. 42 patients with GBM and 16 patients with malignant glioma who had received concurrent radiation and temozolomide therapies, and adjuvant temozolomide were enrolled at first relapse. The 6-month progression-free survival rate was 7.7\% for GBM cohort and $25 \%$ for patients with malignant glioma. Overall radiographic response rate was $24 \%$ (18\% for GBM and $44 \%$ for malignant glioma). The median PFS was 24 weeks for patients with malignant glioma (95\% CI, 5 to 31 weeks) and 12 weeks for patients with GBM (95\% CI, 8 to 16 weeks). A total of 14 patients $(25 \%)$ were removed from the study for toxicity, on average less than 2 months from treatment initiation. This study suggested Aflibercept monotherapy had moderate toxicity and minimal evidence of single-agent activity in unselected patients with recurrent malignant glioma.

\subsubsection{Cediranib}

Several inhibitors for VEGFR tyrosine kinase have shown significant antiangiogenic and antitumor activity in preclinical GBM models $[83 ; 84 ; 85 ; 86 ; 87 ; 88]$, which may also enhance cytotoxic therapy [89; 90; 91]. In addition, several these agents are undergoing evaluation in phase I/II clinical trials, but only cediranib has advanced to phase III investigation. In an initial phase II study of single-agent cediranib $(45 \mathrm{mg} / \mathrm{d}), 27 \%$ of patients with recurrent malignant glioma exhibited a radiographic response and a 6-month PFS was $26 \%$. In addition, cediranib induced rapid normalization of tumor vasculature, including decreased diameter of microvessels and diminished permeability, which reversed after cediranib interruption. Adverse events including hypertension and fatigue were observed, and nearly half of the patients required a dose reduction or interruption of therapy because of its toxicity [92].

\subsubsection{Mechanisms of resistance to antiangiogenic therapy}

Although antiangiogenic therapies prolong PFS of GBM patients, further progression of disease is inevitable. Progression of tumors under antiangiogenic therapy cannot often be treated successfully thereafter, and most patients die of the disease within a few months. In the cediranib study, serum levels of the proangiogenic factors bFGF, stromal-derived factor 1 (SDF1), and soluble VEGFR2 increased at the time of failure [93]. The alternative proangiogenic pathways depends on these angiogenic factors may drive angiogenesis in the setting of VEGFR inhibition. Furthermore, for many gliomas, particularly malignant gliomas, there is often little evidence for vascular proliferation. As the individual infiltrating tumor cell tends to grow along normal cerebral vasculature, and thus there is no need for tumor-associated angiogenesis. Indeed, there is at least a theoretical concern that inhibition of angiogenesis in malignant glioma may prevent the formation bulky tumor but has little effect on sparsely infiltrative GBM cells results in little impact on OS of patients. Early clinical and radiographic observations of patients treated with bevacizumab suggest that this may be the case $[94 ; 95]$. Another concern is recent laboratory evidence that suggests that inhibition of VEGF may actually increase invasiveness of tumor cells [96]. The infiltrative tumor cells are often responsible for relapse leading to the death of patients. 
Combination of antiangiogenic and anti-invasion therapy may delay disease progression. Studies of co-administration of cediranib (pan-VEGFR inhibitor) with cilengitide (integrin inhibitor) and bevacizumab (neutralizing VEGF antibody) with dasatinib (PDGFR $\beta$ inhibitor) are ongoing. Another potential mechanism of resistance to antiangiogenic therapies involves increased PDGF signaling. PDGF stabilizes neovasculature by recruiting pericytes and facilitating pericyte-endothelial cell interactions [97]. Preclinical data suggest that dual VEGFR/PDGFR inhibition potentiates antiangiogenic efficacy and reduces resistance to therapy [98], and this approach is currently being evaluated in clinical trials.

\subsection{4. c-MET}

Aberrant signaling by hepatocyte growth factor (HGF) and its receptor MET has been observed in various tumors including GBM, and potential involvement in tumorigenesis and metastasis has been reported [99]. Recently c-MET overexpression was detected in 18 (29\%) of 62 GBM with shorter median survival durations than those of little or no expression of cMET (median durations of survival, 11.7 vs 14.3 months) [100].

\subsubsection{AMG102 and PF02341066}

Inhibitors of HGF or c-MET have shown preclinical activity against GBM cell lines [99]. The anti-HGF antibody AMG102 enhanced TMZ-induced inhibition of growth of GBM cell line in vitro and in vivo as xenografts [101]. However, phase II trial suggests AMG 102 monotherapy did not significantly suppress tumor growth of recurrent GBM [102]. PF02341066, an orally available ATP-competitive inhibitor of c-MET inhibited growth and c-MET phosphorylation of GBM in preclinical studies [103]. This molecule is currently under clinical investigation in patients with advanced cancers.

\subsubsection{PI3K and related pathways}

PI3K plays a role in intracellular signaling pathways regulating in cell survival, growth, and proliferation. Activated PI3K is recruited to the cell membrane where it mediates signaling after activation of receptor tyrosine kinases. Downstream targets include AKT for cell proliferation and survival; glycogen synthase kinase-3 (GSK-3) for regulation of cMYC; and mammalian target of rapamycin (mTOR) for regulation of protein synthesis and negative regulator of PI3K. In malignant glioma, PI3K/Akt/mTOR signaling is frequently activated because of the stimulation of receptor tyrosine kinases as EGFR, PDGFR, and mesenchymal-epithelial transition factor (MET), mutation of oncogenic PI3K subunits, and/or loss of PTEN tumor suppressor activity. Therefore inhibiting the PI3K pathway may have therapeutic potential.

\subsubsection{NVP-BEZ235 and Enzastaurin}

NVP-BEZ235, an orally available kinase inhibitor for PDK1, mTOR, and PI3K, induced G1 arrest of a GBM cell line in vitro and enhanced TMZ efficacy in vivo [104]. NVP-BEZ235 treatment is currently in phase I trials involving patients with solid tumors. 
Enzastaurin, a PKC/PI3K/AKT inhibitor, suppressed proliferation and induced apoptosis via a caspase-dependent mechanism in GBM cells in vitro [105]. In vivo models showed that enzastaurin combined with radiotherapy synergistically reduced tumor volume, radiation-induced satellite tumor formation, upregulation of VEGF expression, neovascularization, and GSK-3 $\beta$ phosphorylation [106]. In phase II study of enzastaurin in patients with recurrent heavily pretreated GBM showed that objective radiographic responses occurred in $25 \%$ of patients [107]. The subsequent phase III trial comparing lomustine and enzastaurin at first or second recurrence was the first phase III trial to evaluate a targeted therapy for recurrent GBM. Enzastaurin was well tolerated and had a better hematologic toxicity profile but did not have superior efficacy compared with lomustine in patients with recurrent GBM [108].

\subsubsection{SRC and SRC-Family kinases}

SRC and SRC-Family Kinases (SFKs) are frequently activated in GBM [109] frequently due to their overexpression [110]. SRC and SFKs are promiscuous regulators of multiple signaling pathways for cell proliferation, adhesion, migration, and invasion, which are important processes in tumor invasion and metastasis.

\subsubsection{Dasatinib}

Dasatinib is a potent inhibitor of SRC and SFKs and has been approved for the treatment of certain types of leukemia on the basis of inactivation of BCR-ABL [111]. Dasatinib also inhibits c-KIT and PDGFR [112]. In GBM cells, dasatinib inhibited migration and induced autophagy, resulted in cell death which was enhanced by combination with TMZ [111; 113]. Dasatinib inhibited invasion, promoted tumor regression, induced apoptosis in EGFRvIII-expressing GBM, and enhanced the activity of anti-EGFR antibodies [111]. Trials of dasatinib are ongoing in GBM and several solid tumors. A phase I/II trial involving patients with newly diagnosed GBM is assessing dasatinib combined with radiotherapy and concomitant TMZ, followed by adjuvant dasatinib plus TMZ. Trials of dasatinib for treatment of recurrent GBM include a phase II trial of dasatinib monotherapy, a phase I trial of dasatinib in combination with erlotinib, and a randomized phase I/II trial of dasatinib in combination with CCNU that has started its phase I component with patients who have recurrent GBM.

\subsubsection{Integrin}

Integrin plays key roles regulating cell adhesion, migration, and invasion. In addition to a role for matrix-cell contact, integrin also activate intracellular signals including SRC-dependent pathway. In various tumors, integrin has an established role in metastasis and angiogenesis [114]. Therefore, targeting integrin function may have potential for treating GBM. 


\begin{tabular}{|c|c|c|c|}
\hline PRIMARY TARGET & AGENT & OTHER TARGETS & MECHANISM OF ACTION \\
\hline \multirow[t]{7}{*}{ EGFR } & Gefitinib (ZD1839) & & TKI \\
\hline & Erlotinib (OSI-774) & & TKI \\
\hline & Lapatinib (GW-572016) & HER-2 & TKI \\
\hline & PF-00299804 & HER-2, HER-4 & TKI (irreversible) \\
\hline & BIBW2992 & HER-2, HER-4 & TKI (irreversible) \\
\hline & Cetuximab & & Monoclonal antibody \\
\hline & Nimotuzumab & & Monoclonal antibody \\
\hline EGFRvIlI & CDX110 & & Vaccine \\
\hline PDGFR- $\alpha$ & IMC3G3 & & Monoclonal antibody \\
\hline \multirow[t]{3}{*}{ PDGFR- $\beta$} & Imatinib & BCR/Abl, c-Kit & TKI \\
\hline & Dasatinib & Src, BCR/Abl, c-Kit, ephrin A2 & TKI \\
\hline & Tandutinib (MLN518) & Flt3, c-Kit & TKI \\
\hline \multirow[t]{2}{*}{ VEGF-A } & Aflibercept (VEGF Trap) & VEGF-B, PIGF & Soluble decoy receptor \\
\hline & Bevacizumab & & Monoclonal antibody \\
\hline \multirow[t]{6}{*}{ VEGFR-2 } & Cediranib (AZD2171) & $\begin{array}{l}\text { All VEGFR subtypes, PDGFR- } \beta \\
\text {,c-Kit }\end{array}$ & Adnectin \\
\hline & CT-322 & All VEGFR subtypes & TKI \\
\hline & Pazopanib & $\begin{array}{l}\text { All VEGFR subtypes, PDGFR- } \alpha \\
\text { And } \beta, c-K i t\end{array}$ & TKI \\
\hline & Sorafenib & $\begin{array}{l}\text { VEGFR-3, B-Raf, PDGFR- } \beta \\
\text {, c-Kit, Ras, p } 38 \alpha\end{array}$ & TKI \\
\hline & Sunitinib & PDGFR- $\beta$, Flt3, c-Kit & TKI \\
\hline & Vandetanib (ZD6474) & EGFR & TKI \\
\hline c-Met & XL-184 & VEGFR & TKI \\
\hline $\mathrm{HGF} / \mathrm{SF}$ & 17-AAG & & Monoclonal antibody \\
\hline PI3K & $\mathrm{XL765}$ & mTOR & STKI \\
\hline PKC & Enzastaurin (LY31761) & & STKI \\
\hline \multirow[t]{4}{*}{ mTOR } & Sirolimus (rapamycin) & & mTOR inhibitor \\
\hline & Everolimus (RAD001) & & mTOR inhibitor \\
\hline & Temsirolimus (CCI-779) & & mTOR inhibitor \\
\hline & Ridaforolimus (AP23573) & & mTOR inhibitor \\
\hline SRC & Dasatinib & & TKI \\
\hline Integrins & Cilengitide (EMD121974) & & Synthetic RGD peptide \\
\hline \multirow[t]{3}{*}{ HDAC } & Vorinostat (SAHA) & & HDAC inhibitor \\
\hline & Valproic acid & & HDAC inhibitor \\
\hline & LBH589 & & HDAC inhibitor \\
\hline
\end{tabular}

Table 2. Targeted molecular agents currently in clinical development for high-grade glioma TKI indicates tyrosine kinase inhibitor; SAHA, suberoylanilide hydroxamic acid; RGD, arginine-glycine-aspartate; STKI. serine-threnoine kinase inhibitor; PKC, protein kinase $C$. 


\subsubsection{Cilengitide}

Cilengitide is a specific $\alpha \mathrm{V}$ integrin inhibitor in clinical development. In a phase I/IIa trial, cilengitide combined with the current standard of therapy in patients with newly diagnosed GBM was well tolerated, with 6-month PFS as 69\%. Methylation of promoter of O6-methylguanine-DNA methyltransferase (MGMT) predicts a higher likelihood of achieving 6-month PFS, as shown by increases in the durations of PFS and OS to 13.4 months and 23.2 months, respectively, compared with 3.4 and 13.1 months for patients without MGMT promoter methylation [115]. On the basis of these findings, a similar regimen is being compared with radiotherapy/TMZ alone in the phase III CENTRIC trial in patients with newly diagnosed GBM with hypermethylated MGMT promoter. In a phase IIa study of recurrent GBM, cilengitide monotherapy was well tolerated but was largely inactive (6-month PFS rate, 15\%); long-term disease stabilization was seen in a small subset of patients: $10 \%$ were progression free for 12 months, and $5 \%$ were progression free for 24 months [116].

\subsubsection{Histone deacetylase inhibitor}

Histone deacetylases (HDACs) are involved in multiple processes to lead malignant phenotype of glioma including maintanance of stemness, angiogenesis, and resistance to DNA damage.

\subsubsection{Vorinostat}

Vorinostat is an orally available inhibitor of class I and II HDAC approved for advance cutaneous T cell lymphoma. In a phase II study of recurrent GBM, vorinostat monotherapy was well tolerated and had modest clinical activity (6-month PFS rate, 15.2\%; median OS duration, 5.7 months) [117]. Vorinostat is currently being evaluated for use in newly diagnosed and recurrent GBM as a combination therapy.

\section{Conclusion}

Although TMZ prolonged the survival of GBM patients, GBM are still immortal disease with extremely poor prognosis because of acquisition of TMZ resistance. Therefore, other therapeutic agents which suppress MGMT expression or attenuate TMZ resistance are highly desired. As the efficacy of single agent of targeted molecular therapy seems to be limited, combination therapy should be evaluated since multi-pathway is involved in the chemoresistance in GBM. An 'tailor-made' selection of chemotherapeutic agents for each GBM patients based on molecular analysis is essential to obtain maximum efficacy of chemotherapeutic agents. 


\section{Author details}

Shinji Kohsaka ${ }^{1}$ and Shinya Tanaka ${ }^{1,2}$

*Address all correspondence to: tanaka@med.hokudai.ac.jp

1 Department of Cancer Pathology, Hokkaido University Graduate School of Medicine, Sapporo, Japan

2 Department of Translational Pathology, Hokkaido University Graduate School of Medicine, Sapporo, Japan

\section{References}

[1] E.G. Van Meir, C.G. Hadjipanayis, A.D. Norden, H.K. Shu, P.Y. Wen, J.J. Olson. Exciting new advances in neuro-oncology: the avenue to a cure for malignant glioma. CA Cancer J Clin. May-Jun 2010;60(3):166-193.

[2] L. Liu, S.L. Gerson. Targeted modulation of MGMT: clinical implications. Clin Cancer Res. Jan 15 2006;12(2):328-331.

[3] R. Stupp, W.P. Mason, M.J. van den Bent, et al. Radiotherapy plus concomitant and adjuvant temozolomide for glioblastoma. N Engl J Med. Mar 10 2005;352(10):987-996.

[4] S.C. Schold, Jr., T.P. Brent, E. von Hofe, et al. O6-alkylguanine-DNA alkyltransferase and sensitivity to procarbazine in human brain-tumor xenografts. J Neurosurg. Apr 1989;70(4):573-577.

[5] S.L. Gerson, J.E. Trey, K. Miller, N.A. Berger. Comparison of O6-alkylguanine-DNA alkyltransferase activity based on cellular DNA content in human, rat and mouse tissues. Carcinogenesis. May 1986;7(5):745-749.

[6] M. Citron, R. Decker, S. Chen, et al. O6-methylguanine-DNA methyltransferase in human normal and tumor tissue from brain, lung, and ovary. Cancer Res. Aug 15 1991;51(16):4131-4134.

[7] W.J. Washington, R.S. Foote, W.C. Dunn, W.M. Generoso, S. Mitra. Age-dependent modulation of tissue-specific repair activity for 3-methyladenine and O6-methylguanine in DNA in inbred mice. Mech Ageing Dev. Apr 1989;48(1):43-52.

[8] M.E. Hegi, A.C. Diserens, T. Gorlia, et al. MGMT gene silencing and benefit from temozolomide in glioblastoma. N Engl J Med. Mar 10 2005;352(10):997-1003.

[9] M. Weller, J. Felsberg, C. Hartmann, et al. Molecular predictors of progression-free and overall survival in patients with newly diagnosed glioblastoma: a prospective translational study of the German Glioma Network. J Clin Oncol. Dec 1 2009;27(34): 5743-5750. 
[10] [10]P. Das, T. Puri, P. Jha, et al. A clinicopathological and molecular analysis of glioblastoma multiforme with long-term survival. J Clin Neurosci. Jan 2011;18(1):66-70.

[11] I. Lavon, D. Fuchs, D. Zrihan, et al. Novel mechanism whereby nuclear factor kappaB mediates DNA damage repair through regulation of $\mathrm{O}(6)$-methylguanine-DNAmethyltransferase. Cancer Res. Sep 15 2007;67(18):8952-8959.

[12] A.E. Pegg. Repair of O(6)-alkylguanine by alkyltransferases. Mutat Res. Apr 2000;462(2-3):83-100.

[13] T. Grombacher, S. Mitra, B. Kaina. Induction of the alkyltransferase (MGMT) gene by DNA damaging agents and the glucocorticoid dexamethasone and comparison with the response of base excision repair genes. Carcinogenesis. Nov 1996;17(11): 2329-2336.

[14] T. Biswas, C.V. Ramana, G. Srinivasan, et al. Activation of human O6-methylguanine-DNA methyltransferase gene by glucocorticoid hormone. Oncogene. Jan 14 1999;18(2):525-532.

[15] A. Natsume, D. Ishii, T. Wakabayashi, et al. IFN-beta down-regulates the expression of DNA repair gene MGMT and sensitizes resistant glioma cells to temozolomide. Cancer Res. Sep 1 2005;65(17):7573-7579.

[16] D. Bocangel, S. Sengupta, S. Mitra, K.K. Bhakat. p53-Mediated down-regulation of the human DNA repair gene O6-methylguanine-DNA methyltransferase (MGMT) via interaction with Sp1 transcription factor. Anticancer Res. Oct 2009;29(10): 3741-3750.

[17] V. Vielhauer, M. Sarafoff, P. Gais, H.M. Rabes. Cell type-specific induction of O6-alkylguanine-DNA alkyltransferase mRNA expression in rat liver during regeneration, inflammation and preneoplasia. J Cancer Res Clin Oncol. Oct 2001;127(10):591-602.

[18] K.S. Srivenugopal, X.H. Yuan, H.S. Friedman, F. Ali-Osman. Ubiquitination-dependent proteolysis of O6-methylguanine-DNA methyltransferase in human and murine tumor cells following inactivation with O6-benzylguanine or 1,3-bis(2-chloroethyl)-1nitrosourea. Biochemistry. Jan 30 1996;35(4):1328-1334.

[19] V. Ramakrishnan, D. Kushwaha, D.C. Koay, et al. Post-transcriptional regulation of $\mathrm{O}(6)$-methylguanine-DNA methyltransferase MGMT in glioblastomas. Cancer Biomark. 2011;10(3-4):185-193.

[20] J.A. Quinn, A. Desjardins, J. Weingart, et al. Phase I trial of temozolomide plus O6benzylguanine for patients with recurrent or progressive malignant glioma. J Clin Oncol. Oct 1 2005;23(28):7178-7187.

[21] M. Ranson, M.R. Middleton, J. Bridgewater, et al. Lomeguatrib, a potent inhibitor of O6-alkylguanine-DNA-alkyltransferase: phase I safety, pharmacodynamic, and pharmacokinetic trial and evaluation in combination with temozolomide in patients with advanced solid tumors. Clin Cancer Res. Mar 1 2006;12(5):1577-1584. 
[22] O. Khan, M.R. Middleton. The therapeutic potential of O6-alkylguanine DNA alkyltransferase inhibitors. Expert Opin Investig Drugs. Oct 2007;16(10):1573-1584.

[23] J.A. Quinn, S.X. Jiang, D.A. Reardon, et al. Phase II trial of temozolomide plus o6benzylguanine in adults with recurrent, temozolomide-resistant malignant glioma. J Clin Oncol. Mar 10 2009;27(8):1262-1267.

[24] A. Yoshino, A. Ogino, K. Yachi, et al. Effect of IFN-beta on human glioma cell lines with temozolomide resistance. Int J Oncol. Jul 2009;35(1):139-148.

[25] S. Kohsaka, L. Wang, K. Yachi, et al. STAT3 inhibition overcomes temozolomide resistance in glioblastoma by downregulating MGMT expression. Mol Cancer Ther. Jun 2012;11(6):1289-1299.

[26] D.P. Cahill, K.K. Levine, R.A. Betensky, et al. Loss of the mismatch repair protein MSH6 in human glioblastomas is associated with tumor progression during temozolomide treatment. Clin Cancer Res. Apr 1 2007;13(7):2038-2045.

[27] S. Yip, J. Miao, D.P. Cahill, et al. MSH6 mutations arise in glioblastomas during temozolomide therapy and mediate temozolomide resistance. Clin Cancer Res. Jul 15 2009;15(14):4622-4629.

[28] J.B. Tang, D. Svilar, R.N. Trivedi, et al. N-methylpurine DNA glycosylase and DNA polymerase beta modulate BER inhibitor potentiation of glioma cells to temozolomide. Neuro Oncol. May 2011;13(5):471-486.

[29] S. Agnihotri, A.S. Gajadhar, C. Ternamian, et al. Alkylpurine-DNA-N-glycosylase confers resistance to temozolomide in xenograft models of glioblastoma multiforme and is associated with poor survival in patients. J Clin Invest. Jan 3 2012;122(1): 253-266.

[30] N. Kondo, A. Takahashi, E. Mori, et al. FANCD1/BRCA2 plays predominant role in the repair of DNA damage induced by ACNU or TMZ. PLoS One. 2011;6(5):e19659.

[31] P. Pyrko, A.H. Schonthal, F.M. Hofman, T.C. Chen, A.S. Lee. The unfolded protein response regulator GRP78/BiP as a novel target for increasing chemosensitivity in malignant gliomas. Cancer Res. Oct 15 2007;67(20):9809-9816.

[32] Y. Gao, A. Fotovati, C. Lee, et al. Inhibition of Y-box binding protein-1 slows the growth of glioblastoma multiforme and sensitizes to temozolomide independent O6methylguanine-DNA methyltransferase. Mol Cancer Ther. Dec 2009;8(12):3276-3284.

[33] N. Gaspar, L. Marshall, L. Perryman, et al. MGMT-independent temozolomide resistance in pediatric glioblastoma cells associated with a PI3-kinase-mediated HOX/stem cell gene signature. Cancer Res. Nov 15 2010;70(22):9243-9252.

[34] Z. Jiang, N. Pore, G.J. Cerniglia, et al. Phosphatase and tensin homologue deficiency in glioblastoma confers resistance to radiation and temozolomide that is reversed by the protease inhibitor nelfinavir. Cancer Res. May 1 2007;67(9):4467-4473. 
[35] Comprehensive genomic characterization defines human glioblastoma genes and core pathways. Nature. Oct 23 2008;455(7216):1061-1068.

[36] D.W. Parsons, S. Jones, X. Zhang, et al. An integrated genomic analysis of human glioblastoma multiforme. Science. Sep 26 2008;321(5897):1807-1812.

[37] W. Xu, H. Yang, Y. Liu, et al. Oncometabolite 2-hydroxyglutarate is a competitive inhibitor of alpha-ketoglutarate-dependent dioxygenases. Cancer Cell. Jan 18 2011;19(1):17-30.

[38] L. Dang, D.W. White, S. Gross, et al. Cancer-associated IDH1 mutations produce 2hydroxyglutarate. Nature. Dec 10 2009;462(7274):739-744.

[39] L. Frederick, X.Y. Wang, G. Eley, C.D. James. Diversity and frequency of epidermal growth factor receptor mutations in human glioblastomas. Cancer Res. Mar 1 2000;60(5):1383-1387.

[40] P. Kleihues, H. Ohgaki. Primary and secondary glioblastomas: from concept to clinical diagnosis. Neuro Oncol. Jan 1999;1(1):44-51.

[41] D.M. Peereboom, D.R. Shepard, M.S. Ahluwalia, et al. Phase II trial of erlotinib with temozolomide and radiation in patients with newly diagnosed glioblastoma multiforme. J Neurooncol. May 2010;98(1):93-99.

[42] M.D. Prados, S.M. Chang, N. Butowski, et al. Phase II study of erlotinib plus temozolomide during and after radiation therapy in patients with newly diagnosed glioblastoma multiforme or gliosarcoma. J Clin Oncol. Feb 1 2009;27(4):579-584.

[43] J.F. de Groot, M.R. Gilbert, K. Aldape, et al. Phase II study of carboplatin and erlotinib (Tarceva, OSI-774) in patients with recurrent glioblastoma. J Neurooncol. Oct 2008;90(1):89-97.

[44] D.A. Reardon, A. Desjardins, J.J. Vredenburgh, et al. Phase 2 trial of erlotinib plus sirolimus in adults with recurrent glioblastoma. J Neurooncol. Jan 2010;96(2):219-230.

[45] M.J. van den Bent, A.A. Brandes, R. Rampling, et al. Randomized phase II trial of erlotinib versus temozolomide or carmustine in recurrent glioblastoma: EORTC brain tumor group study 26034. J Clin Oncol. Mar 10 2009;27(8):1268-1274.

[46] B. Thiessen, C. Stewart, M. Tsao, et al. A phase I/II trial of GW572016 (lapatinib) in recurrent glioblastoma multiforme: clinical outcomes, pharmacokinetics and molecular correlation. Cancer Chemother Pharmacol. Jan 2010;65(2):353-361.

[47] B. Neyns, J. Sadones, E. Joosens, et al. Stratified phase II trial of cetuximab in patients with recurrent high-grade glioma. Ann Oncol. Sep 2009;20(9):1596-1603.

[48] D.A. Haas-Kogan, M.D. Prados, T. Tihan, et al. Epidermal growth factor receptor, protein kinase B/Akt, and glioma response to erlotinib. J Natl Cancer Inst. Jun 15 2005;97(12):880-887. 
[49] I.K. Mellinghoff, M.Y. Wang, I. Vivanco, et al. Molecular determinants of the response of glioblastomas to EGFR kinase inhibitors. N Engl J Med. Nov 10 2005;353(19):2012-2024.

[50] T. Ozawa, C.W. Brennan, L. Wang, et al. PDGFRA gene rearrangements are frequent genetic events in PDGFRA-amplified glioblastomas. Genes Dev. Oct 1 2010;24(19): 2205-2218.

[51] S.M. Wilhelm, L. Adnane, P. Newell, A. Villanueva, J.M. Llovet, M. Lynch. Preclinical overview of sorafenib, a multikinase inhibitor that targets both Raf and VEGF and PDGF receptor tyrosine kinase signaling. Mol Cancer Ther. Oct 2008;7(10):3129-3140.

[52] C. Yu, B.B. Friday, J.P. Lai, et al. Cytotoxic synergy between the multikinase inhibitor sorafenib and the proteasome inhibitor bortezomib in vitro: induction of apoptosis through Akt and c-Jun NH2-terminal kinase pathways. Mol Cancer Ther. Sep 2006;5(9):2378-2387.

[53] E.P. Jane, D.R. Premkumar, I.F. Pollack. Coadministration of sorafenib with rottlerin potently inhibits cell proliferation and migration in human malignant glioma cells. J Pharmacol Exp Ther. Dec 2006;319(3):1070-1080.

[54] D. Hagerstrand, G. Hesselager, S. Achterberg, et al. Characterization of an imatinibsensitive subset of high-grade human glioma cultures. Oncogene. Aug 10 2006;25(35):4913-4922.

[55] M. Holdhoff, K.A. Kreuzer, C. Appelt, et al. Imatinib mesylate radiosensitizes human glioblastoma cells through inhibition of platelet-derived growth factor receptor. Blood Cells Mol Dis. Mar-Apr 2005;34(2):181-185.

[56] A. Desjardins, J.A. Quinn, J.J. Vredenburgh, et al. Phase II study of imatinib mesylate and hydroxyurea for recurrent grade III malignant gliomas. J Neurooncol. May 2007;83(1):53-60.

[57] P.Y. Wen, W.K. Yung, K.R. Lamborn, et al. Phase I/II study of imatinib mesylate for recurrent malignant gliomas: North American Brain Tumor Consortium Study 99-08. Clin Cancer Res. Aug 15 2006;12(16):4899-4907.

[58] E. Raymond, A.A. Brandes, C. Dittrich, et al. Phase II study of imatinib in patients with recurrent gliomas of various histologies: a European Organisation for Research and Treatment of Cancer Brain Tumor Group Study. J Clin Oncol. Oct 1 2008;26(28): 4659-4665.

[59] D.A. Reardon, G. Dresemann, S. Taillibert, et al. Multicentre phase II studies evaluating imatinib plus hydroxyurea in patients with progressive glioblastoma. Br J Cancer. Dec 15 2009;101(12):1995-2004.

[60] G. Dresemann, M. Weller, M.A. Rosenthal, et al. Imatinib in combination with hydroxyurea versus hydroxyurea alone as oral therapy in patients with progressive pretreated glioblastoma resistant to standard dose temozolomide. J Neurooncol. Feb 2010;96(3):393-402. 
[61] W. Wick, M. Weller, M. Weiler, T. Batchelor, A.W. Yung, M. Platten. Pathway inhibition: emerging molecular targets for treating glioblastoma. Neuro Oncol. Jun 2011;13(6):566-579.

[62] K.H. Plate, H.D. Mennel. Vascular morphology and angiogenesis in glial tumors. Exp Toxicol Pathol. May 1995;47(2-3):89-94.

[63] E. Bullitt, D.A. Reardon, J.K. Smith. A review of micro- and macrovascular analyses in the assessment of tumor-associated vasculature as visualized by MR. Neuroimage. 2007;37 Suppl 1:S116-119.

[64] S.K. Hobbs, W.L. Monsky, F. Yuan, et al. Regulation of transport pathways in tumor vessels: role of tumor type and microenvironment. Proc Natl Acad Sci U S A. Apr 14 1998;95(8):4607-4612.

[65] S. Morikawa, P. Baluk, T. Kaidoh, A. Haskell, R.K. Jain, D.M. McDonald. Abnormalities in pericytes on blood vessels and endothelial sprouts in tumors. Am J Pathol. Mar 2002;160(3):985-1000.

[66] P. Baluk, S. Morikawa, A. Haskell, M. Mancuso, D.M. McDonald. Abnormalities of basement membrane on blood vessels and endothelial sprouts in tumors. Am J Pathol. Nov 2003;163(5):1801-1815.

[67] T. Inai, M. Mancuso, H. Hashizume, et al. Inhibition of vascular endothelial growth factor (VEGF) signaling in cancer causes loss of endothelial fenestrations, regression of tumor vessels, and appearance of basement membrane ghosts. Am J Pathol. Jul 2004;165(1):35-52.

[68] W.S. Kamoun, C.D. Ley, C.T. Farrar, et al. Edema control by cediranib, a vascular endothelial growth factor receptor-targeted kinase inhibitor, prolongs survival despite persistent brain tumor growth in mice. J Clin Oncol. May 20 2009;27(15):2542-2552.

[69] H.S. Friedman, M.D. Prados, P.Y. Wen, et al. Bevacizumab alone and in combination with irinotecan in recurrent glioblastoma. J Clin Oncol. Oct 1 2009;27(28):4733-4740.

[70] T.N. Kreisl, L. Kim, K. Moore, et al. Phase II trial of single-agent bevacizumab followed by bevacizumab plus irinotecan at tumor progression in recurrent glioblastoma. J Clin Oncol. Feb 10 2009;27(5):740-745.

[71] D. Zagzag, H. Zhong, J.M. Scalzitti, E. Laughner, J.W. Simons, G.L. Semenza. Expression of hypoxia-inducible factor 1alpha in brain tumors: association with angiogenesis, invasion, and progression. Cancer. Jun 1 2000;88(11):2606-2618.

[72] N.O. Schmidt, M. Westphal, C. Hagel, et al. Levels of vascular endothelial growth factor, hepatocyte growth factor/scatter factor and basic fibroblast growth factor in human gliomas and their relation to angiogenesis. Int J Cancer. Feb 19 1999;84(1): $10-18$. 
[73] Y.H. Zhou, F. Tan, K.R. Hess, W.K. Yung. The expression of PAX6, PTEN, vascular endothelial growth factor, and epidermal growth factor receptor in gliomas: relationship to tumor grade and survival. Clin Cancer Res. Aug 15 2003;9(9):3369-3375.

[74] J.R. Flynn, L. Wang, D.L. Gillespie, et al. Hypoxia-regulated protein expression, patient characteristics, and preoperative imaging as predictors of survival in adults with glioblastoma multiforme. Cancer. Sep 1 2008;113(5):1032-1042.

[75] N. Ferrara, K.J. Hillan, H.P. Gerber, W. Novotny. Discovery and development of bevacizumab, an anti-VEGF antibody for treating cancer. Nat Rev Drug Discov. May 2004;3(5):391-400.

[76] E.T. Wong, K.R. Hess, M.J. Gleason, et al. Outcomes and prognostic factors in recurrent glioma patients enrolled onto phase II clinical trials. J Clin Oncol. Aug 1999;17(8):2572-2578.

[77] F.M. Iwamoto, H.A. Fine. Bevacizumab for malignant gliomas. Arch Neurol. Mar 2010;67(3):285-288.

[78] J.J. Vredenburgh, A. Desjardins, J.E. Herndon, 2nd, et al. Bevacizumab plus irinotecan in recurrent glioblastoma multiforme. J Clin Oncol. Oct 20 2007;25(30):4722-4729.

[79] A. Desjardins, D.A. Reardon, J.E. Herndon, 2nd, et al. Bevacizumab plus irinotecan in recurrent WHO grade 3 malignant gliomas. Clin Cancer Res. Nov 1 2008;14(21): 7068-7073.

[80] K.R. Lamborn, W.K. Yung, S.M. Chang, et al. Progression-free survival: an important end point in evaluating therapy for recurrent high-grade gliomas. Neuro Oncol. Apr 2008;10(2):162-170.

[81] J. Holash, S. Davis, N. Papadopoulos, et al. VEGF-Trap: a VEGF blocker with potent antitumor effects. Proc Natl Acad Sci U S A. Aug 20 2002;99(17):11393-11398.

[82] J.F. de Groot, K.R. Lamborn, S.M. Chang, et al. Phase II study of aflibercept in recurrent malignant glioma: a North American Brain Tumor Consortium study. J Clin Oncol. Jul 1 2011;29(19):2689-2695.

[83] S. de Bouard, P. Herlin, J.G. Christensen, et al. Antiangiogenic and anti-invasive effects of sunitinib on experimental human glioblastoma. Neuro Oncol. Oct 2007;9(4): 412-423.

[84] F. Hilberg, G.J. Roth, M. Krssak, et al. BIBF 1120: triple angiokinase inhibitor with sustained receptor blockade and good antitumor efficacy. Cancer Res. Jun 15 2008;68(12):4774-4782.

[85] J.N. Rich, S. Sathornsumetee, S.T. Keir, et al. ZD6474, a novel tyrosine kinase inhibitor of vascular endothelial growth factor receptor and epidermal growth factor receptor, inhibits tumor growth of multiple nervous system tumors. Clin Cancer Res. Nov 15 2005;11(22):8145-8157. 
[86] J.J. Yiin, B. Hu, P.A. Schornack, et al. ZD6474, a multitargeted inhibitor for receptor tyrosine kinases, suppresses growth of gliomas expressing an epidermal growth factor receptor mutant, EGFRvIII, in the brain. Mol Cancer Ther. Apr 2010;9(4):929-941.

[87] F. Yang, C. Brown, R. Buettner, et al. Sorafenib induces growth arrest and apoptosis of human glioblastoma cells through the dephosphorylation of signal transducers and activators of transcription 3. Mol Cancer Ther. Apr 2010;9(4):953-962.

[88] Y. Zhang, F. Guessous, A. Kofman, D. Schiff, R. Abounader. XL-184, a MET, VEGFR-2 and RET kinase inhibitor for the treatment of thyroid cancer, glioblastoma multiforme and NSCLC. IDrugs. Feb 2010;13(2):112-121.

[89] A.J. Schueneman, E. Himmelfarb, L. Geng, et al. SU11248 maintenance therapy prevents tumor regrowth after fractionated irradiation of murine tumor models. Cancer Res. Jul 15 2003;63(14):4009-4016.

[90] V. Damiano, D. Melisi, C. Bianco, et al. Cooperative antitumor effect of multitargeted kinase inhibitor ZD6474 and ionizing radiation in glioblastoma. Clin Cancer Res. Aug 1 2005;11(15):5639-5644.

[91] Q. Zhou, P. Guo, J.M. Gallo. Impact of angiogenesis inhibition by sunitinib on tumor distribution of temozolomide. Clin Cancer Res. Mar 1 2008;14(5):1540-1549.

[92] T.T. Batchelor, D.G. Duda, E. di Tomaso, et al. Phase II study of cediranib, an oral pan-vascular endothelial growth factor receptor tyrosine kinase inhibitor, in patients with recurrent glioblastoma. J Clin Oncol. Jun 10 2010;28(17):2817-2823.

[93] T.T. Batchelor, A.G. Sorensen, E. di Tomaso, et al. AZD2171, a pan-VEGF receptor tyrosine kinase inhibitor, normalizes tumor vasculature and alleviates edema in glioblastoma patients. Cancer Cell. Jan 2007;11(1):83-95.

[94] F.M. Iwamoto, L.E. Abrey, K. Beal, et al. Patterns of relapse and prognosis after bevacizumab failure in recurrent glioblastoma. Neurology. Oct 13 2009;73(15):1200-1206.

[95] A.D. Norden, J. Drappatz, A. Muzikansky, et al. An exploratory survival analysis of anti-angiogenic therapy for recurrent malignant glioma. J Neurooncol. Apr 2009;92(2):149-155.

[96] M. Paez-Ribes, E. Allen, J. Hudock, et al. Antiangiogenic therapy elicits malignant progression of tumors to increased local invasion and distant metastasis. Cancer Cell. Mar 3 2009;15(3):220-231.

[97] P. Guo, B. Hu, W. Gu, et al. Platelet-derived growth factor-B enhances glioma angiogenesis by stimulating vascular endothelial growth factor expression in tumor endothelia and by promoting pericyte recruitment. Am J Pathol. Apr 2003;162(4): 1083-1093.

[98] R. Erber, A. Thurnher, A.D. Katsen, et al. Combined inhibition of VEGF and PDGF signaling enforces tumor vessel regression by interfering with pericyte-mediated endothelial cell survival mechanisms. FASEB J. Feb 2004;18(2):338-340. 
[99] L. Toschi, P.A. Janne. Single-agent and combination therapeutic strategies to inhibit hepatocyte growth factor/MET signaling in cancer. Clin Cancer Res. Oct 1 2008;14(19):5941-5946.

[100] D.S. Kong, S.Y. Song, D.H. Kim, et al. Prognostic significance of c-Met expression in glioblastomas. Cancer. Jan 1 2009;115(1):140-148.

[101] H.T. Jun, J. Sun, K. Rex, et al. AMG 102, a fully human anti-hepatocyte growth factor/ scatter factor neutralizing antibody, enhances the efficacy of temozolomide or docetaxel in U-87 MG cells and xenografts. Clin Cancer Res. Nov 15 2007;13(22 Pt 1): 6735-6742.

[102] P.Y. Wen, D. Schiff, T.F. Cloughesy, et al. A phase II study evaluating the efficacy and safety of AMG 102 (rilotumumab) in patients with recurrent glioblastoma. Neuro Oncol. Apr 2011;13(4):437-446.

[103] S. Yamazaki, J. Skaptason, D. Romero, et al. Pharmacokinetic-pharmacodynamic modeling of biomarker response and tumor growth inhibition to an orally available cMet kinase inhibitor in human tumor xenograft mouse models. Drug Metab Dispos. Jul 2008;36(7):1267-1274.

[104] S.M. Maira, F. Stauffer, J. Brueggen, et al. Identification and characterization of NVPBEZ235, a new orally available dual phosphatidylinositol 3-kinase/mammalian target of rapamycin inhibitor with potent in vivo antitumor activity. Mol Cancer Ther. Jul 2008;7(7):1851-1863.

[105] J. Rieger, D. Lemke, G. Maurer, et al. Enzastaurin-induced apoptosis in glioma cells is caspase-dependent and inhibited by BCL-XL. J Neurochem. Sep 2008;106(6): 2436-2448.

[106] G. Tabatabai, B. Frank, A. Wick, et al. Synergistic antiglioma activity of radiotherapy and enzastaurin. Ann Neurol. Feb 2007;61(2):153-161.

[107] T.N. Kreisl, S. Kotliarova, J.A. Butman, et al. A phase I/II trial of enzastaurin in patients with recurrent high-grade gliomas. Neuro Oncol. Feb 2010;12(2):181-189.

[108] W. Wick, V.K. Puduvalli, M.C. Chamberlain, et al. Phase III study of enzastaurin compared with lomustine in the treatment of recurrent intracranial glioblastoma. J Clin Oncol. Mar 1 2010;28(7):1168-1174.

[109] J. Du, P. Bernasconi, K.R. Clauser, et al. Bead-based profiling of tyrosine kinase phosphorylation identifies SRC as a potential target for glioblastoma therapy. Nat Biotechnol. Jan 2009;27(1):77-83.

[110] K.V. Lu, S. Zhu, A. Cvrljevic, et al. Fyn and SRC are effectors of oncogenic epidermal growth factor receptor signaling in glioblastoma patients. Cancer Res. Sep 1 2009;69(17):6889-6898. 
[111] M. Brave, V. Goodman, E. Kaminskas, et al. Sprycel for chronic myeloid leukemia and Philadelphia chromosome-positive acute lymphoblastic leukemia resistant to or intolerant of imatinib mesylate. Clin Cancer Res. Jan 15 2008;14(2):352-359.

[112] L.J. Lombardo, F.Y. Lee, P. Chen, et al. Discovery of N-(2-chloro-6-methyl- phenyl)-2(6-(4-(2-hydroxyethyl)- piperazin-1-yl)-2-methylpyrimidin-4- ylamino)thiazole-5-carboxamide (BMS-354825), a dual Src/Abl kinase inhibitor with potent antitumor activity in preclinical assays. J Med Chem. Dec 30 2004;47(27):6658-6661.

[113] V. Milano, Y. Piao, T. LaFortune, J. de Groot. Dasatinib-induced autophagy is enhanced in combination with temozolomide in glioma. Mol Cancer Ther. Feb 2009;8(2):394-406.

[114] J.S. Desgrosellier, D.A. Cheresh. Integrins in cancer: biological implications and therapeutic opportunities. Nat Rev Cancer. Jan 2010;10(1):9-22.

[115] R. Stupp, M.E. Hegi, B. Neyns, et al. Phase I/Ila study of cilengitide and temozolomide with concomitant radiotherapy followed by cilengitide and temozolomide maintenance therapy in patients with newly diagnosed glioblastoma. J Clin Oncol. Jun 1 2010;28(16):2712-2718.

[116] D.A. Reardon, K.L. Fink, T. Mikkelsen, et al. Randomized phase II study of cilengitide, an integrin-targeting arginine-glycine-aspartic acid peptide, in recurrent glioblastoma multiforme. J Clin Oncol. Dec 1 2008;26(34):5610-5617.

[117] E. Galanis, K.A. Jaeckle, M.J. Maurer, et al. Phase II trial of vorinostat in recurrent glioblastoma multiforme: a north central cancer treatment group study. J Clin Oncol. Apr 20 2009;27(12):2052-2058. 